\title{
Overcoming cetuximab resistance in Ewing's sarcoma by inhibiting lactate dehydrogenase- $A$
}

\author{
JIAXIN FU, HAN JIANG, CHENXUAN WU, YI JIANG，LIANPING XIAO and YONGGANG TIAN \\ Department of Orthopedics, Tianjin Third Central Hospital, Tianjin 300170, P.R. China
}

Received January 26, 2015; Accepted December 14, 2015

DOI: $10.3892 / \mathrm{mmr} .2016 .5290$

\begin{abstract}
Ewing's sarcoma, the second most common type of malignant bone tumor, generally occurs in children and young adults. The current treatment of Ewing's sarcoma comprises systemic anti-cancer chemotherapy with complete surgical resection. However, the majority of patients with Ewing's sarcoma develop resistance to chemotherapy. The present study revealed an oncogenic role of lactate dehydrogenase-A (LDHA) in the resistance of Ewing's sarcoma to cetuximab. LDHA was shown to be upregulated at the protein and mRNA level in cetuximab-resistant Ewing's sarcoma tissues and a cell line. In addition, a link between LDHA-induced glycolysis and cetuximab resistance in Ewing's sarcoma cells was revealed. Of note, inhibition of LDHA by either small interfering RNA or LDHA inhibitor oxamate significantly re-sensitized cetuximab-resistant cells to cetuximab. Combined treatment with LDHA inhibitor and cetuximab synergistically reduced the viability of cetuximab-resistant cells through the suppression of LDHA. The present study revealed a novel mechanism of cetuximab resistance from the perspective of cancer-cell metabolism and provided a sensitization approach, which may aid in the development of anti-chemoresistance strategies for the treatment of cetuximab-resistant Ewing's sarcoma.
\end{abstract}

\section{Introduction}

Ewing's sarcoma is a primary malignant bone cancer type that mainly affects children and adolescents, while it rarely occurs in adults over the age of 30 years (1-3). It is the second most common type of bone cancer in children with a male/female ratio of 1.6:1 $(1,2)$. The currently used treatment of Ewing's sarcoma consists of a combination of systemic anti-cancer chemotherapy and complete surgical resection (3-5). However, the majority of patients with Ewing's sarcoma develop

Correspondence to: Dr Han Jiang, Department of Orthopedics, Tianjin Third Central Hospital, 83 Jintang Road, Hedong, Tianjin 300170, P.R. China

E-mail: hanjiang1958@163.com

Key words: Ewing's sarcoma, cetuximab resistance, chemotherapy, lactate dehydrogenase-A, Warburg effect resistance to chemotherapy (5). The underlying mechanisms of the chemoresistance of Ewing's sarcoma have been identified to include enhanced drug-efflux pump activity, upregulation of DNA repair mechanisms, changes in the intracellular metabolic pathway (6), the aberrant expression of microRNA (7) and impairment of apoptosis (6). Therefore, novel treatment strategies with reduced chemoresistance as well as methods to inhibit the abovementioned mechanisms of chemoresistance are required, while a relatively low toxicity to surrounding normal tissues is also essential.

Epidermal growth factor receptor (EGFR), an abundant transmembrane glycoprotein of the tyrosine kinase growth factor receptor family $(8,9)$, is expressed in numerous types of normal human tissues; the activation of this proto-oncogene by ligand binding to its extra-cellular domain results in the activation of downstream cellular signals that regulate cell growth, resistance to anti-cancer drugs, promotion of tumor invasion and angiogenesis $(9,10)$. Cetuximab (CTX) is an anti-cancer drug with a binding affinity to EGFR greater than that of its natural ligand EGF, thereby blocking the ligand-induced activation of EGFR. In addition, cetuximab stimulates the internalization of EGFR thereby removing it from the cell surface and any possible ligand interaction (11).

Lactate dehydrogenase-A (LDHA) is one of the metabolic key enzymes controlling the conversion of pyruvate to lactate as part of the cellular glycolytic process (12). Studies have reported that LDHA has a key role in tumor growth, chemoresistance and tumor metastasis of numerous types of cancer (13). LDHA has been identified as a direct target of the c-myc and EGFR oncogenes $(12,14)$. In addition, LDHA is regulated by hypoxia-inducible factor $(\mathrm{HIF}-1 \alpha)(14)$. The present study explored the correlation between LDHA expression and CTX resistance in human Ewing's sarcoma, revealing that LDHA is highly expressed in CTX-resistant Ewing's sarcoma tissues and a cell line. Inhibition of LDHA enhanced the sensitivity of Ewing's sarcoma cells to CTX. The present study revealed a novel mechanism of cetuximab resistance from the perspective of cancer cell metabolism.

\section{Materials and methods}

Cell culture and Ewing's sarcoma patient samples. The A673 human Ewing's sarcoma cell line was purchased from the American Type Culture Collection (Manassas, VA, USA). Cells were cultured in Dulbecco's modified Eagle's medium 
(Mediatech Inc., Manassas, VA, USA) with $10 \%$ fetal bovine serum in an incubator containing a humidified atmosphere with $5 \% \mathrm{CO}_{2}$ at $37^{\circ} \mathrm{C}$. Eight cetuximab-resistant and two cetuximab-sensitive primary Ewing's sarcoma tissue samples were used in the present study, which were in this project, which were obtained from male and female patients (mean age, 24.2; age range, 15-43 years; male/female gender ratio, 1.4/1) undergoing surgery for Ewing's sarcoma between January 2013 and October 2014 at the Department of Orthopedics, Tianjin Third Central Hospital (Tianjin, China). The CTX-resistant samples were obtained from patients who had a negative response to CTX treatment and the CTX-sensitive samples from patients who had a positive a response to CTX treatment. All samples were stored in liquid nitrogen until analysis. Tumors were obtained following a protocol approved by the Ethics Committee of the Department of Orthopedics, Tianjin Third Central Hospital (Tianjin, China). All patients provided written informed consent.

Antibodies and reagents. The following antibodies were used in the present study: LDHA (cat no. sc-130327; Santa Cruz Biotechnology, Inc., Dallas, TX, USA) and GAPDH (Scat no. sc-365062; Santa Cruz Biotechnology, Inc.). Oxamate was purchased from Sigma-Aldrich (St. Louis, MO, USA) and CTX was purchased from ImClone Systems (Bridgewater, NJ, USA).

Generation of a CTX resistant cell line. A673 human Ewing's sarcoma cells were treated with CTX with stepwise dose increases of up to $20 \mu \mathrm{g} / \mathrm{ml}$ under standard cell culture conditions for selection of resistant cells. After successive treatments in continuous culture for four months, several resistant cell clones were developed from the parental cell line. CTX resistant pooled clones were used for all subsequent experiments in the present study. The resistant cells were re-selected by CTX treatments each month.

Western blot analysis. Cells were lysed in lysis buffer containing $50 \mathrm{mmol} / \mathrm{l}$ Tris- $\mathrm{HCl}$ (pH 7.4), $150 \mathrm{mmol} / \mathrm{l} \mathrm{NaCl}$, $0.5 \% \mathrm{NP}-40,50 \mathrm{mmol} / 1 \mathrm{NaF}, 1 \mathrm{mmol} / 1 \mathrm{Na}_{3} \mathrm{VO}_{4}, 1 \mathrm{mmol} / \mathrm{l}$ phenylmethylsulfonyl fluoride, $25 \mathrm{mg} / \mathrm{ml}$ aprotinin and $25 \mathrm{mg} / \mathrm{ml}$ leupeptin (Bio-Rad Laboratories, Inc., Hercules, CA, USA) and kept on ice for $15 \mathrm{~min}$. Following centrifugation at $12,000 \mathrm{x} \mathrm{g}$ for $15 \mathrm{~min}$ at $4^{\circ} \mathrm{C}$, the supernatants were collected and the protein concentration was determined using the Pierce Coomassie Plus colorimetric protein assay (Thermo Fisher Scientific, Inc.). Subsequently, equal amounts of protein (50 $\mu \mathrm{g}$ for each lane) were subjected to 4-20\% SDS-PAGE gradient gel (Bio-Rad Laboratories, Inc.) and transferred onto a nitrocellulose membrane (Bio-Rad Laboratories, Inc.). The membrane was blocked by $4 \%$ non-fat dry milk for $1 \mathrm{~h}$ at room temperature prior to the incubation. The membrane was incubated with primary LDHA mouse monoclonal anti-human IgG1 (cat. no., sc-130327; dilution, 1:1,000) and/or GAPDH mouse monoclonal IgG1 antibodies (cat. no., sc-365062; dilution, $1: 1,000)$ overnight at $4^{\circ} \mathrm{C}$ in phosphate-buffered saline (PBS) containing 5\% non-fat dry milk. Both primary antibodies were obtained from Santa Cruz Biotechnology, Inc., Dallas, TX, USA. Following extensive washing with PBS, membranes were incubated with a horse anti-mouse $\operatorname{IgG}$ (heavy and light chain) horseradish peroxidase-conjugated secondary antibody (cat. no., 7074; dilution, 1:2,000; Cell Signaling Technology,
Inc., Danvers, MA, USA). The antigen-antibody complexes were then visualized using an Enhanced Chemiluminescence Detection kit (GE Healthcare, Little Chalfont, UK). The images were developed using ChemiDoc ${ }^{\mathrm{TM}}$ Touch Gel Imaging System (1708370; Bio-Rad Laboratories, Inc., Hercules, CA, USA). The quantification of the blots was performed using ImageJ software.

cDNA preparation and reverse-transcription quantitative polymerase chain reaction $(R T-q P C R)$ analysis. Cells and tissues were homogenized and total RNA was extracted using an RNeasy mini kit (Qiagen, Hilden Germany), which was simultaneously subjected to DNase digestion performed using the High Capacity cDNA Reverse Transcription kit (Applied Biosystems; Thermo Fisher Scientific, Inc., Waltham, MA, USA). Real-time qPCR was then performed to amplify the obtained cDNA; the PCR mixture contained TaqMan Gene Expression Assay primers and probes specific for LDHA were supplied by Applied Biosystems and were as follows: 5'-ATC TTGACCTACGTGGCTTGGA-3' forward and 5'-CCATAC AGGCACACTGGAATCTC-3' reverse. Ribosomal 18S RNA, was used as an internal control and the primers used were as follows: 5'-CTACCACATCCAAGGAAGCA-3' forward and 5'-TTTTTCGTCACTACCTCCCCG-3' reverse (Applied Biosystems). PCR amplifications were performed in a final reaction volume of $10 \mu \mathrm{l}$ containing $0.5 \mu \mathrm{l}$ of the primers and probes mix, 5.5 $\mu \mathrm{l}$ TaqMan Universal PCR Master Mix (Applied Biosystems) and 4.5 $\mu \mathrm{g}$ cDNA solution diluted at 1:10. The thermocycling conditions were as follows: One cycle of $50^{\circ} \mathrm{C}$ for $2 \mathrm{~min}$ and initial denaturation for $10 \mathrm{~min}$ at $95^{\circ} \mathrm{C}$, followed by 40 cycles of denaturation $\left(15 \mathrm{sec}\right.$ at $\left.95^{\circ} \mathrm{C}\right)$ and annealing extension $\left(1 \mathrm{~min}\right.$ at $\left.60^{\circ} \mathrm{C}\right)$. The Step 1 Plus Real-Time PCR Systems Thermocycler (Applied Biosystems) was used for all reactions. RTqPCR was performed in triplicate with at least two repetitions. The quantification cycle $(\mathrm{Cq})$ of $18 \mathrm{~S}$ ribosomal RNA was used to determine the $\triangle \mathrm{Cq}$ value for mRNA expression. The formula $2^{(-\Delta \Delta \mathrm{Cq})}$ was used to calculate the relative mRNA expression (15).

Plasmid DNA and small interfering (si)RNA transfections. Transfections were performed using the Oligofectamine Transfection reagent (Invitrogen; Thermo Fisher Scientific, Inc.) according to the manufacturer's instructions. Overexpression vector for wild type LDAH (cat no. RC209378) was purchased from Origene (Cambridge, UK). siRNA oligonucleotides for LDHA were purchased from Sigma-Aldrich, with a scrambled siRNA (Sigma-Aldrich) used as a control. Following $48 \mathrm{~h}$ hours of transfection, whole-cell lysates were prepared for subsequent analysis.

Glucose consumption assay. Cells were seeded in six-well plates at $5 \times 10^{5}$ cells per well in $3 \mathrm{ml}$ phenol red-free, low-glucose, low-serum cell culture medium (Thermo Fisher Scientific, Inc.). Following treatment with the cell culture medium, a $50-\mu 1$ aliquot of the conditioned medium was collected from each well and diluted with $950 \mu$ l distilled water (1:20). The glucose concentration in the diluted medium was measured using the Glucose Assay kit (Sigma-Aldrich) according to the manufacturer's instructions and the protocol of a previous study (14). Glucose consumption was calculated by subtracting the concentration of glucose remaining in the medium at the 


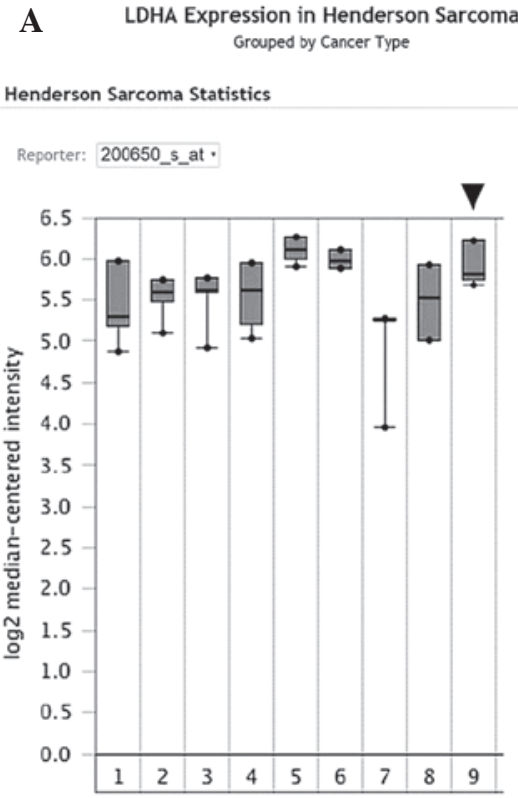

B

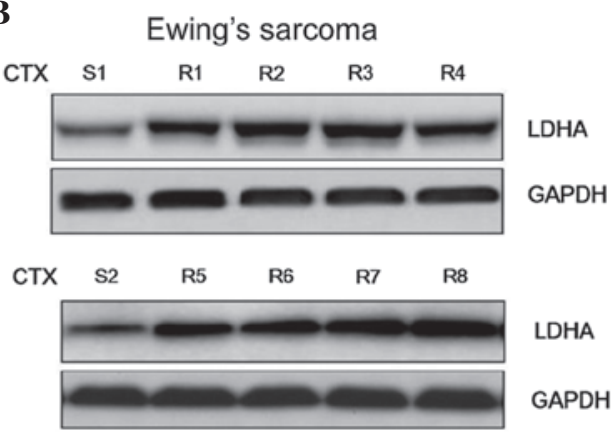

C

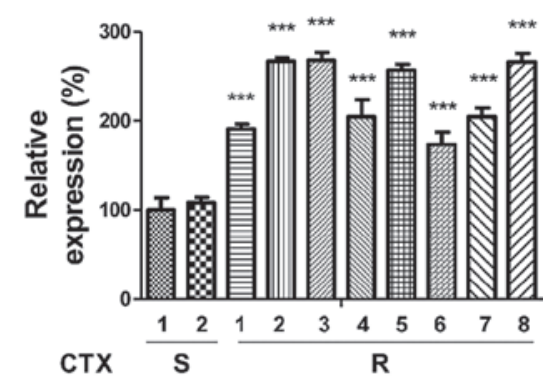

Legend

1. Alveolar Rhabdomyosarcoma (4)

2. Chondroblastoma (4)

3. Chondromyxoid Fibroma (4)

4. Chondrosarcoma (7)

5. Chordoma (4)

6. Dedifferentiated Chondrosarcoma (3)

7. Deep Fibromatosis/Desmoid Tumor (5)

8. Embryonal Rhabdomyosarcoma (3)

9. Ewing's Sarcoma (5)

Figure 1. LDHA is upregulated in CTX-resistant Ewing's sarcoma samples. (A) LDHA expression statistics in sarcoma types sourced from oncomine.com by Henderson et al $(16,17)$. The expression of LDHA from different types of bone tumor tissue was analyzed using previously published microarray data. (B) Western blot analysis of LDHA in CTX-sensitive and -resistant human Ewing's sarcoma samples. GAPDH was used as a loading control. (C) mRNA levels of LDHA in CTX-sensitive and -resistant human Ewing's sarcoma samples determined by polymerase chain reaction analysis. Values are expressed as the mean \pm standard error of three independent experiments. ${ }^{* * *} \mathrm{P}<0.001$ vs. S. CTX, cetuximab; LDHA, lactate dehydrogenase-A; S, sensitive; R, resistant.

indicated times from the concentration of glucose present in fresh cell culture medium and results were normalized to the amount of total protein compared with that in the control group.

Lactate production assay. Cells were seeded in six-well plates at $5 \times 10^{5}$ cells per well in $3 \mathrm{ml}$ phenol red-free, low-glucose, low-serum cell culture medium. After the designated treatments, a $50 \mu \mathrm{l}$ aliquot of the conditioned medium was collected from each well and diluted with $950 \mu \mathrm{l}$ distilled water (1:20). The lactate concentration in the diluted medium was measured using the Lactate Assay kit from BioVision Inc. (Cambridge, UK) according to the protocol of a previous study (14). Results were normalized to the amount of total protein compared with that in the control group.

Cell survival assay. Cell survival was measured using a colorimetric ELISA kit (Cell Death Detection ELISA; Roche Diagnostics Corp., Basel, Switzerland), which quantitatively measures cytoplasmic histone-associated DNA fragments (mononucleosomes and oligonucleosomes). The procedure was performed according to the manufacturer's instructions. The results were verified by trypan blue staining (Bio-Rad Laboratories, Inc. and direct cell counting using a hemotocytometer (Thermo Fisher Scientific, Inc.).

Microarray database analysis. Data on the expression of LDHA in Ewing's sarcoma were retrieved from the Oncomine cancer microarray database (oncomine.org) in analogy with a previous study $(16,17)$. Oncomine contains 65 gene expression datasets comprising $\sim 48$ million gene expression measurements form $>4700$ microarray experiments.

Statistical analysis. The statistical analysis was performed using GraphPad Prism 5.0 (GraphPad Software, Inc., La Jolla,
CA, USA). Student's $t$-test was used for all statistical analyses. Values are expressed as the mean \pm standard error. $\mathrm{P}<0.05$ was considered to indicate a statistically significant difference. All experiments were repeated at least once with reproducible results.

\section{Results}

LDHA is associated with CTX resistance in Ewing's sarcoma. Since dysregulated glycolysis of cancer cells has been studied as a target for overcoming chemoresistance and LDHA is an important key enzyme of glycolysis, the present study explored the link between LDHA and Ewing's sarcoma. Bioinformatics analysis using the oncomine online microarray database revealed that LDHA was upregulated in Ewing's sarcoma (Fig. 1A), indicating that LDHA may act as an oncogene and contribute to chemoresistance. The present study assessed the LDHA expression at the protein levels (Fig. 1B) as well as the mRNA levels (Fig. 1C) in CTX-sensitive and resistant Ewing's sarcoma tissues. As expected, the expression of LDHA the in eight CTX-resistant Ewing's sarcoma tissues was significantly upregulated compared with that in the nonresistant tissues, suggesting that LDHA may be an important biomarker for the clinical diagnosis and treatment of Ewing's sarcoma.

CTX-resistant Ewing's sarcoma cells display an upregulated LDHA expression and glycolysis rate. To assess the underlying mechanisms of the chemoresistance to CTX and to determine any possible links with the dysregulated glycolysis of cancer cells, an isogenic CTX-resistant human Ewing's sarcoma cell line, A673 CTX-R, was generated by incubating parental cells to cetuximab with stepwise dose increases of up to $20 \mu \mathrm{g} / \mathrm{ml}$ in continuous culture for four months. To verify the resistance, A673 CTX-R cells and native A673 cells were treated with 

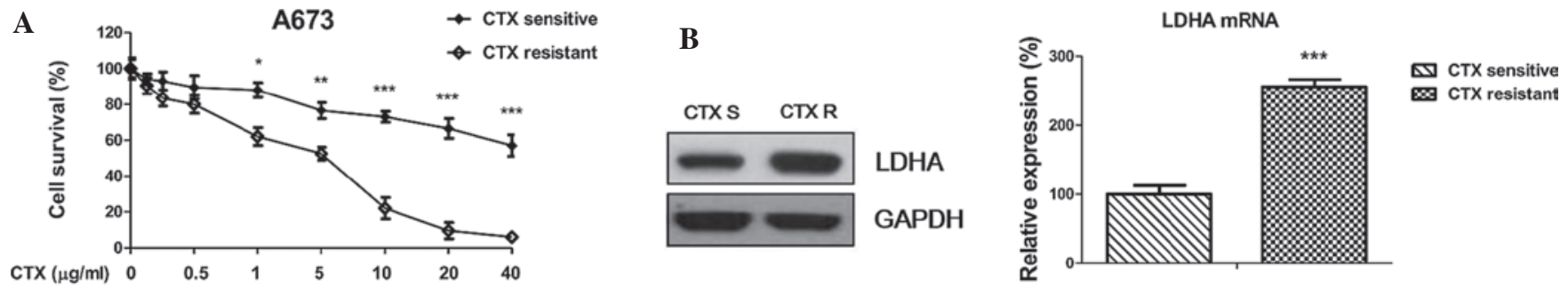

C

Glucose consumption
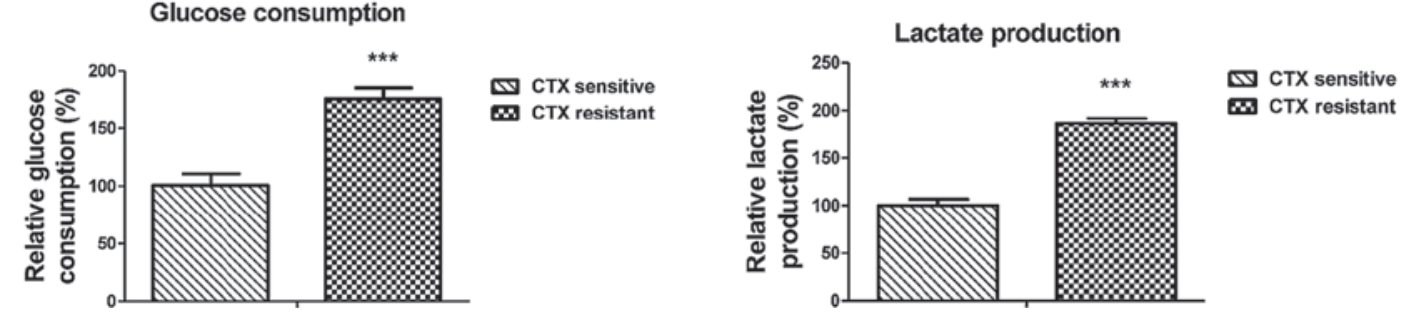

Figure 2. CTX-resistant Ewing's sarcoma cells exhibit increased LDHA expression. (A) Establishment of a CTX-resistant A673 cell line. CTX-sensitive and -resistant cells were treated with CTX at the indicated concentrations for $48 \mathrm{~h}$ and DNA fragmentation in the cell lysates was subsequently assessed using ELISA. (B) LDHA expression in CTX-sensitive and -resistant A673 cells was assessed by western blot (left) and reverse-transcription quantitative polymerase chain reaction (right). GAPDH was used as a loading control/reference gene. (C) Levels of glucose (left) and lactate (right) in the supernatant of CTX-sensitive and -resistant cells following incubation in low-glucose $(1 \mathrm{~g} / 1)$, low-serum $(0.5 \%)$ medium for $24 \mathrm{~h}$. Values are expressed as the mean \pm standard error of three independent experiments. ${ }^{*} \mathrm{P}<0.05 ;{ }^{* *} \mathrm{P}<0.01 ;{ }^{* * *} \mathrm{P}<0.001$ vs. CTX-sensitive. CTX, cetuximab; $\mathrm{S}$, sensitive; R, resistant.

A

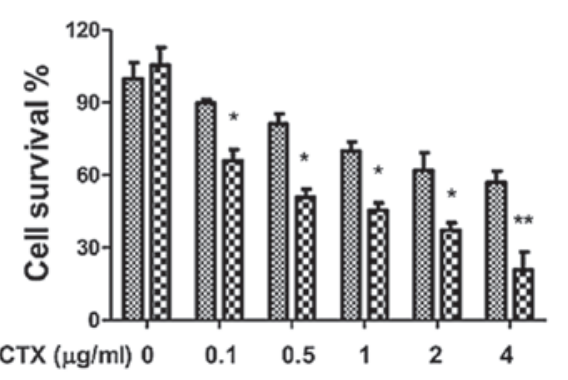

C

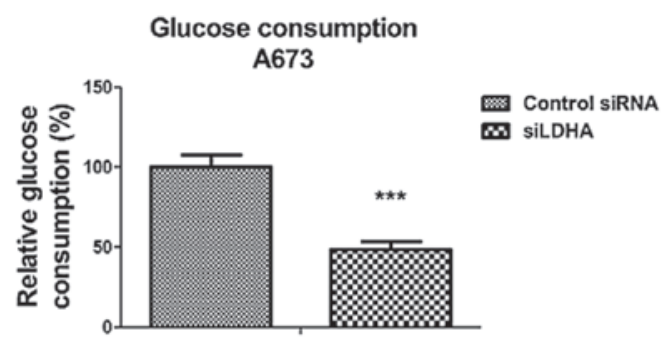

D

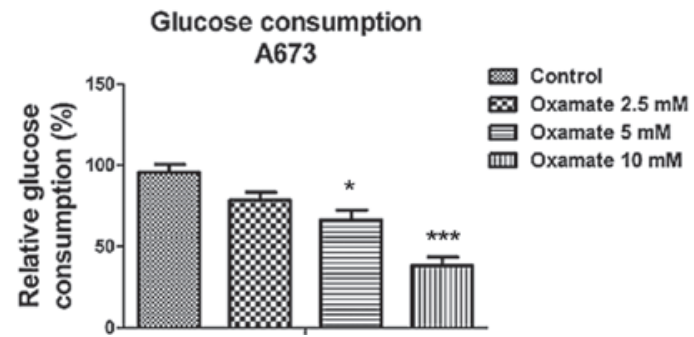

B
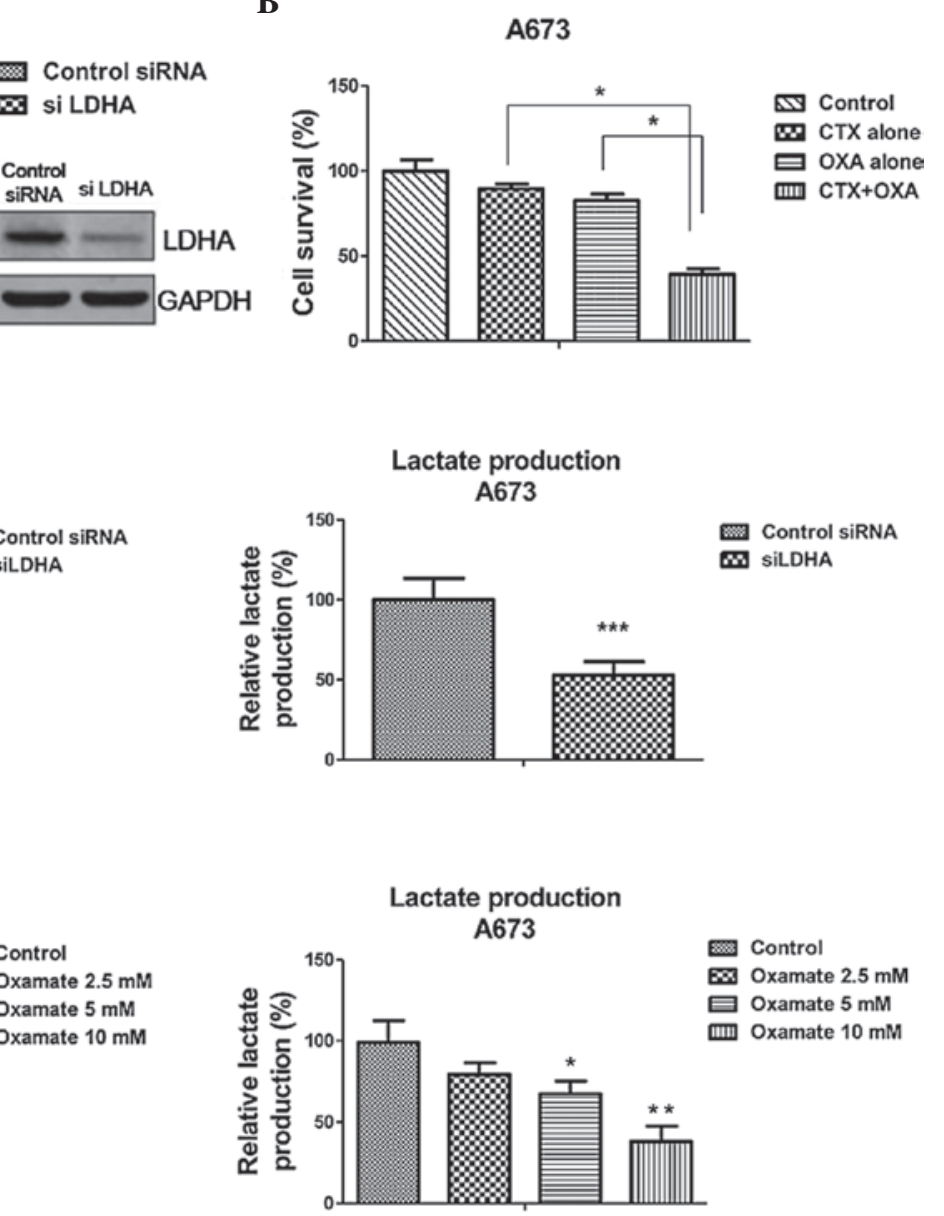

Figure 3. Inhibition of LDHA sensitizes Ewing's sarcoma cells to CTX. (A) LDHA knockdown enhanced the cells' sensitivity to cetuximab. A673 cells were transfected with control siRNA or siLDHA for 48 hours, followed by treatment with cetuximab at the indicated concentrations for $24 \mathrm{~h}$. The cell survival ratio was determined by ELISA of DNA fragments following lysis. (B) A673 cells were transfected with control siRNA or siLDHA for $48 \mathrm{~h}$, and levels of glucose (left) and lactate (right) in the supernatant were determined following incubation in low-glucose $(1 \mathrm{~g} / 1)$, low-serum $(0.5 \%)$ medium for $24 \mathrm{~h}$. (C) A673 cells were treated with CTX alone at $0.5 \mu \mathrm{g} / \mathrm{ml}$, oxamate alone at $2 \mathrm{mM}$ or with their combination for $48 \mathrm{~h}$, followed by the measurement of cell survival using ELISA. (D) A673 cells were treated with oxamate $(2.5,5$ or $10 \mathrm{mM}$ ) for $48 \mathrm{~h}$, and levels of glucose (left) and lactate (right) in the supernatant were determined following incubation in low-glucose $(1 \mathrm{~g} / \mathrm{l})$, low-serum $(0.5 \%)$ medium for $24 \mathrm{~h}$. Values are expressed as the mean \pm standard error of three independent experiments. ${ }^{*} \mathrm{P}<0.05 ;{ }^{* *} \mathrm{P}<0.01 ;{ }^{* * *} \mathrm{P}<0.001$ vs. control. CTX, cetuximab; LDHA, lactate dehydrogenase-A; S, sensitive; R, resistant; siRNA, small interfering RNA. 
A

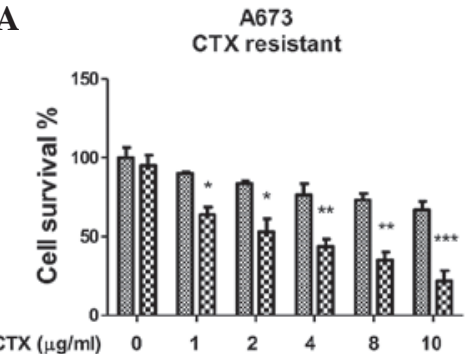

C

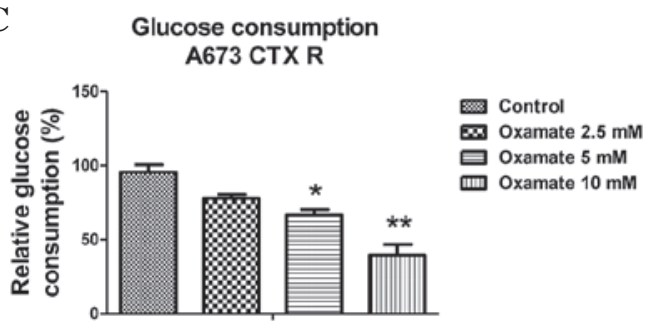

B
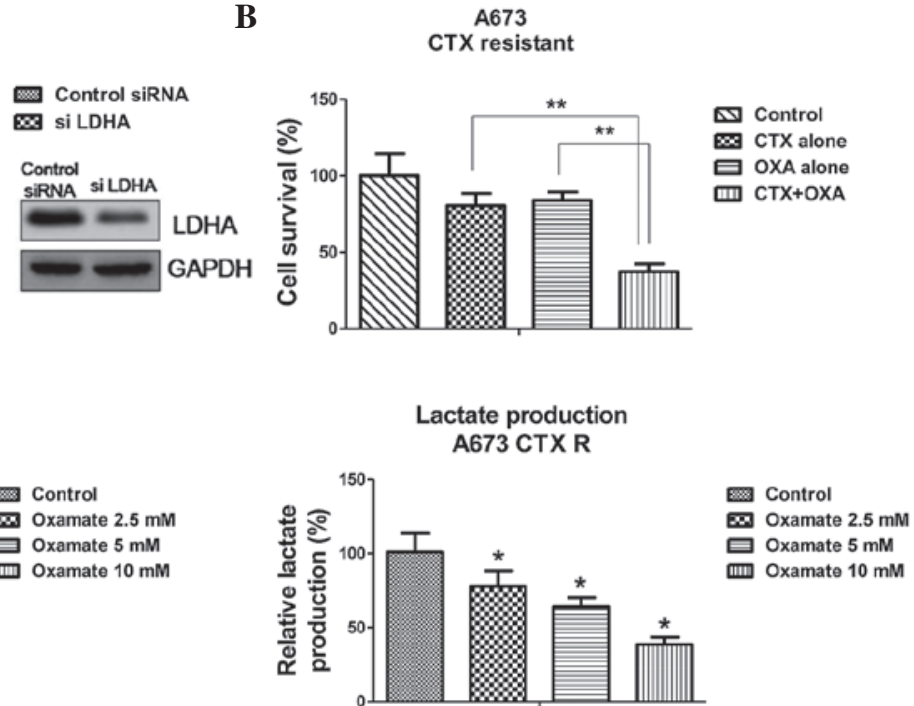

Figure 4. Inhibition of LDHA re-sensitizes CTX-resistant cells. (A) CTX-resistant A673 cells were transfected with control siRNA or siLDHA for 48 h, followed by treatment with CTX at the indicated concentrations for $24 \mathrm{~h}$. The cell survival ratio was determined using ELISA. (B) CTX-resistant A673 cells were treated with CTX alone at $5 \mu \mathrm{g} / \mathrm{ml}$, oxamate alone at $2 \mathrm{mM}$ or with their combination for $48 \mathrm{~h}$, followed by assessment of cell survival using ELISA. (C) CTX-resistant A673 cells were treated with oxamate $(2.5,5$ or $10 \mathrm{mM})$ for $48 \mathrm{~h}$, and levels of glucose (left) and lactate (right) in the supernatant were determined following incubation in low-glucose $(1 \mathrm{~g} / \mathrm{l})$, low-serum $(0.5 \%)$ medium for $24 \mathrm{~h}$. Values are expressed as the mean \pm standard error of three independent experiments. ${ }^{*} \mathrm{P}<0.05 ;{ }^{* *} \mathrm{P}<0.01 ;{ }^{* * *} \mathrm{P}<0.001$ vs. control. CTX, cetuximab; LDHA, lactate dehydrogenase-A; siRNA, small interfering RNA.

CTX under identical experimental conditions (Fig. 2A). As expected, A673 CTX-R cells tolerated significantly higher concentrations of cetuximab compared with CTX-sensitive cells, whose cell survival was significantly impaired by CTX at $5-40 \mu \mathrm{g} / \mathrm{ml}$. Since the abovementioned results revealed that LDHA was linked with CTX resistance in Ewing's sarcoma patient samples, the LDHA levels in CTX-sensitive and resistant cells were determined. In consistency with the results obtained from the tissue samples, LDHA expression in CTX-R A673 cells was upregulated at the protein and mRNA level compared with that in A673 cells (Fig. 2B). To assess the effects of CTX on aerobic glycolysis, glucose consumption and lactate production were directly measured in the supernatants of CTX-sensitive and resistant cells. The results revealed that glucose consumption and lactate production were significantly increased in CTX-resistant cells compared with those in native A673 cells (Fig. 2C), which was an indicator of the dysregulated glycolysis by LDHA in CTX-resistant cells; this aberration may be utilized as a therapeutic target for Ewing's sarcoma.

Inhibition of LDHA enhances sensitivity to CTX. To further elucidate the link between the upregulation of LDHA and the resistance of CTX as well as its targetability for enhancing the efficiency of chemotherapy, a cell survival analysis after subjecting the cells to treatments with CTX and/or knockdown of LDHA by siRNA. LDHA knockdown significantly reduced the survival of A673 cells in the presence of CTX (Fig. 3A). The reversed phenotypes verified that LDHA was upregulated in CTX-resistant Ewing's sarcoma cells. The $\mathrm{IC}_{50}$ of CTX on control-transfected A673 cells was $\sim 4 \mu \mathrm{g} / \mathrm{ml}$, while it was decreased to $\sim 0.5 \mu \mathrm{g} / \mathrm{ml}$ following knockdown of LDHA. Next, the present study investigated the inhibitory effects of a combination of CTX and oxamate (18), which is an inhibitor of LDHA, on the A673 cells. Treatment of
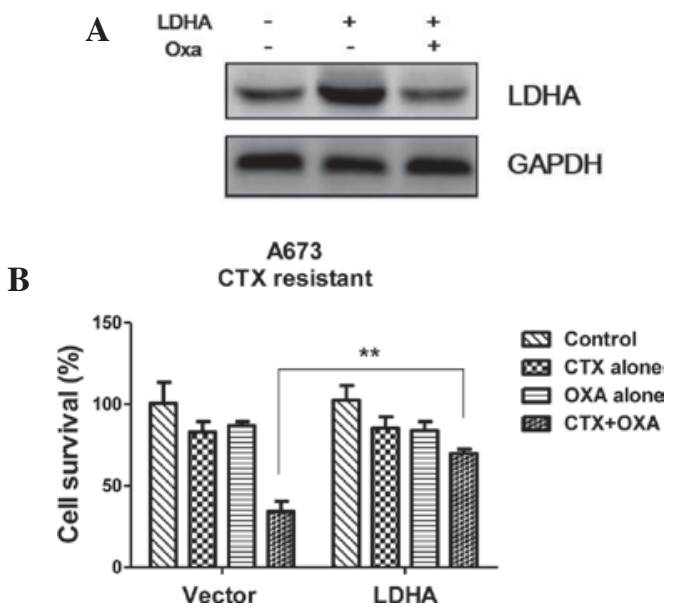

Figure 5. Restoration of LDHA renders CTX-resistant A673 cells resistant to the combination of CTX and OXA. (A) Overexpression of LDHA in OXA-treated and -untreated CTX-resistant A673 cells. (B) CTX-resistant A673 cells were transfected with control or LDHA overexpression vector. At $24 \mathrm{~h}$ following transfection, cells were treated with CTX alone at $5 \mu \mathrm{g} / \mathrm{ml}$, OXA alone at $2 \mathrm{mM}$ or with their combination for $48 \mathrm{~h}$, followed by assessment of cell survival using ELISA. Values are expressed as the mean \pm standard error of three independent experiments. ${ }^{* *} \mathrm{P}<0.01$ vs. control. OXA, oxamate; CTX, cetuximab; LDHA, lactate dehydrogenase-A.

parental A673 cells with CTX alone at $0.5 \mu \mathrm{g} / \mathrm{ml}$ or oxamate alone at $2 \mathrm{mM}$ slightly reduced the cell viability (to 90 and $85 \%$ live cells, respectively), while the combination of CTX and oxamate synergistically reduced the cell viability to a significantly greater extent (38\% live cells) (Fig. 3B), indicating that the inhibition of LDHA either by siRNA or the small-molecule inhibitor oxamate enhanced the chemotherapeutic efficiency of CTX in Ewing's sarcoma. As expected, the glucose consumption and lactate production were significantly decreased following inhibition of LDHA (Fig. 3C and D). 
Synergistic effects of LDHA inhibitor and CTX against $C T X$-resistant Ewing's sarcoma cells. Since the results of the present study demonstrated that LDHA was upregulated in CTX-resistant Ewing's sarcoma cells and that downregulation of LDHA by siRNA or oxamate significantly inhibited the viability of the parental A673 cells, it was hypothesized that inhibition of LDHA in CTX-resistant cells has synergistic effects with CTX. Fig. 4A displays the synergistic inhibitory effects of the combination of LDHA knockdown with CTX in A673-resistant cells. Similarly, CTX combined with oxamate was significantly more potent at inhibiting cell survival compared with either agent alone (Fig. 4B). In consistency with these results, glycolysis was inhibited by either LDHA knockdown as well as oxamate treatment (Fig. 3C and D). In conclusion, CTX and oxamate exhibited a synergistic effect to reduce the viability of CTX-resistant cells, indicating that LDHA inhibition may be an efficient adjuvant in the treatment of Ewing's sarcoma with CTX.

Restoration of LDHA reduces the sensitivity of CTX-resistant A673 cells to CTX and oxamate. To further support the hypothesis that LDHA is associated with resistance to chemotherapy in Ewing's sarcoma, LDHA expression in CTX-resistant cells was restored by transient transfection with LDHA overexpression vector. Western blot analysis showed that the expression of LDHA was enhanced following transfection, while treatment with oxamate reduced LDHA levels to those of untransfected CTX-resistant cells (Fig. 5A). Furthermore, the survival rate of LDHA-overexpressing, CTX-resistant cells was assessed following treatment with CTX alone, oxamate alone or their combination. The chemoresistance of A673 CTX-R cells with restoration of LDHA was shown to be enhanced in the presence of CTX and oxamate combined (Fig. 5B).

\section{Discussion}

The treatment of Ewing's sarcoma requires surgery along with intensive chemotherapy due to the aggressiveness of this cancer type. As resistance to chemotherapy is common in Ewing's sarcoma, the development of strategies to overcome this chemoresistance is urgently required $(1,5)$. The present study compared cetuximab-sensitive and resistant Ewing's sarcoma tissues, indicating that upregulation of LDHA is linked with resistance and may therefore be a target for novel therapeutic strategies. To the best of our knowledge, the present study was the first to report the link between LDHA and CTX resistance in Ewing's sarcoma.

Since cancer cells rely on an uninterrupted supply of energy and nutrients to fuel their unlimited proliferation, they utilize aerobic glycolysis to generate these; for this, they direct the pyruvate pathway towards the production of lactate in the cytosol under catalysis by LDHA, but not through oxidative phosphorylation (19). This unique phenomenon is known as the 'Warburg effect' and leads to an elevated glycolytic flux in cancer cells. It has been reported that CTX reverses the Warburg effect by inhibiting HIF-1-regulated LDHA in cancer cells, indicating that elevated LDHA levels may be associated with resistance to CTX. The present study established a CTX-resistant A673 Ewing's sarcoma cell line and demonstrated elevated levels of LDHA and are a higher glycolytic rate compared with those of their parental cells. Inhibition of LDHA by siRNA or oxamate decreased the glycolysis rate of CTX-sensitive and resistant cells. Furthermore, knockdown or inhibition of LDHA expression in Ewing's sarcoma cells sensitized them to CTX. Consistent with the results of the present study, a previous study reported that upregulated LDHA expression was correlated with Taxol resistance in breast cancer (20). Another recent study reported that inhibition of LDHA by microRNA contributed to the re-sensitization of 5-fluorouracil-resistant colon cancer cells (19). In addition, the present study assessed the potency of the combination of CTX with LDHA inhibitor oxamate against CTX-resistant cells. As expected, the combination of CTX with oxamate showed a synergistic effect to inhibit cell viability. In addition, forced overexpression of LDHA to counteract the oxamate-induced depression of LDHA levels rendered the cells resistant to the combination of CTX and oxamate. The present study suggested that the inhibition of glycolysis is a major mechanism of action of CTX on Ewing's sarcoma. Further experiments are currently underway in our group to explore the underlying mechanisms of CTX-mediated depression of LDHA expression in Ewing's sarcoma cells in more detail. Bioinformatics and proteomics tools will be utilized for the identification of novel targets for overcoming CTX resistance.

\section{Acknowledgements}

The authors would like to thank the following members staff and faculty members of the Department of Orthopedics, Tianjin Third Central Hospital (Tianjin, China): Dr Yi Jiang for providing the patient samples, and Dr Lianping Xiao and Dr Yonggang Tian for their editorial assistance.

\section{References}

1. Iwamoto Y: Diagnosis and treatment of Ewing's sarcoma. Jpn J Clin Oncol 37: 79-89, 2007.

2. Grier HE, Krailo MD, Tarbell NJ, Link MP, Fryer CJ, Pritchard DJ, Gebhardt MC,Dickman PS, Perlman EJ, Meyers PA, et al: Addition of ifosfamide and etoposide to standard chemotherapy for Ewing's sarcoma and primitive neuroectodermal tumor of bone. N Engl J Med 348: 694-701, 2003

3. Miser JS, Krailo MD, Tarbell NJ, Link MP, Fryer CJ, Pritchard DJ, Gebhardt MC, Dickman PS, Perlman EJ, Meyers PA, et al: Treatment of metastatic Ewing's sarcoma or primitive neuroectodermal tumor of bone: Evaluation of combination ifosfamide and etoposide - a Children's cancer group and pediatric oncology group study. J Clin Oncol 22: 2873-2876, 2004.

4. Longtin R: Ewing sarcoma: A miracle drug waiting to happen? J Natl Cancer Inst 95: 1574-1576, 2003.

5. May WA, Grigoryan RS, Keshelava N, Cabral DJ, Christensen LL, Jenabi J, Ji L, Triche TJ, Lawlor ER and Reynolds CP: Characterization and drug resistance patterns of Ewing's sarcoma family tumor cell lines. PLoS One 8: e80060, 2013.

6. Fodale V, Pierobon M, Liotta L and Petricoin E: Mechanism of cell adaptation: When and how do cancer cells develop chemoresistance? Cancer J 17: 89-95, 2011.

7. Iida K, Fukushi J, Matsumoto Y, et al: miR-125b develops chemoresistance in Ewing sarcomaprimitive neuroectodermal tumor. Cancer Cell Int 13: 21, 2013.

8. Bardelli A and Jänne PA: The road to resistance: EGFR mutation and cetuximab. Nat Med 18: 199-200, 2012.

9. Yewale C, Baradia D, Vhora I, Patil S and Misra A: Epidermal growth factor receptor targeting in cancer: A review of trends and strategies. Biomaterials 34: 8690-8707, 2013. 
10. Red Brewer M, Yun CH, Lai D, Lemmon MA, Eck MJ and Pao W: Mechanism for activation of mutated epidermal growth factor receptors in lung cancer. Proc Natl Acad Sci USA 110: E3595-E3604, 2013.

11. Graham J, Muhsin M and Kirkpatrick P: Cetuximab. Nat Rev Drug Discov 3: 549-550, 2004.

12. Fantin VR, St-Pierre J and Leder P: Attenuation of LDH-A expression uncovers a link between glycolysis, mitochondrial physiology and tumor maintenance. Cancer Cell 6: 425-434, 2006.

13. Maftouh M, Avan A, Sciarrillo R, Granchi C, Leon LG, Rani R, Funel N, Smid K, Honeywell R, Boggi U, et al: Synergistic interaction of novel lactate dehydrogenase inhibitors with gemcitabine against pancreatic cancer cells in hypoxia. Br J Cancer 110: 172-182, 2014.

14. Lu H, Li X, Luo Z, Liu J and Fan Z: Cetuximab reverses the Warburg effect by inhibiting HIF-1-regulated LDH-A. Mol Cancer Ther 12: 2187-2199, 2013.

15. Livak KJ and Schmittgen TD: Analysis of relative gene expression data using real-time quantitative PCR and the 2(-Delta Delta C(T)) Method. Methods 25: 402-408, 2001.
16. Rhodes DR, Yu J, Shanker K, Deshpande N, Varambally R, Ghosh D, Barrette T, Pandey A and Chinnaiyan AM: ONCOMINE: A cancer microarray database and integrated data-mining platform. Neoplasia 6: 1-6, 2004.

17. Henderson SR, Guiliano D, Presneau N, McLean S, Frow R, Vujovic S, Anderson J, Sebire N, Whelan J, Athanasou $\mathrm{N}$, et al: A molecular map of mesenchymal tumors. Genome Biol 206: R76, 2005.

18. Zhou M, Zhao Y, Ding Y, Liu H, Liu Z, Fodstad O, Riker AI, Kamarajugadda S, Lu J, Owen LB, et al: Warburg effect in chemosensitivity: Targeting lactate dehydrogenase-A re-sensitizes taxol-resistant cancer cells to taxol. Mol Cancer 9: 33, 2010

19. Vander Heiden MG, Cantley LC and Thompson CB: Understanding the Warburg effect: The metabolic requirements of cell proliferation. Science 324: 1029-1033, 2009.

20. Li X, Zhao H, Zhou X and Song L: Inhibition of lactate dehydrogenase A by microRNA-34a resensitizes colon cancer cells to 5-fluorouracil. Mol Med Rep 11: 577-582, 2015. 